$4 \quad$ Supporting information

5

Figure SI F3: Process diagram

\title{
Production of cyanide using thermal plasma: thermodynamic analysis and experimental specific energy
}

consumption

Figure SI F1 Detailed Thermodynamic profile of C-N-H species (1:2:4), e.g. feed of nitrogen and methane

Figure SI F2: Detailed Thermodynamic profile of $\mathrm{C}-\mathrm{H}-\mathrm{N}$ species same as figure 1 but excluding condensed carbon

Table SI T1: Experimental data and mass balance (Nitrogen and Propane)

Table SI T2: Experimental data and mass balance (Nitrogen and Methane) 


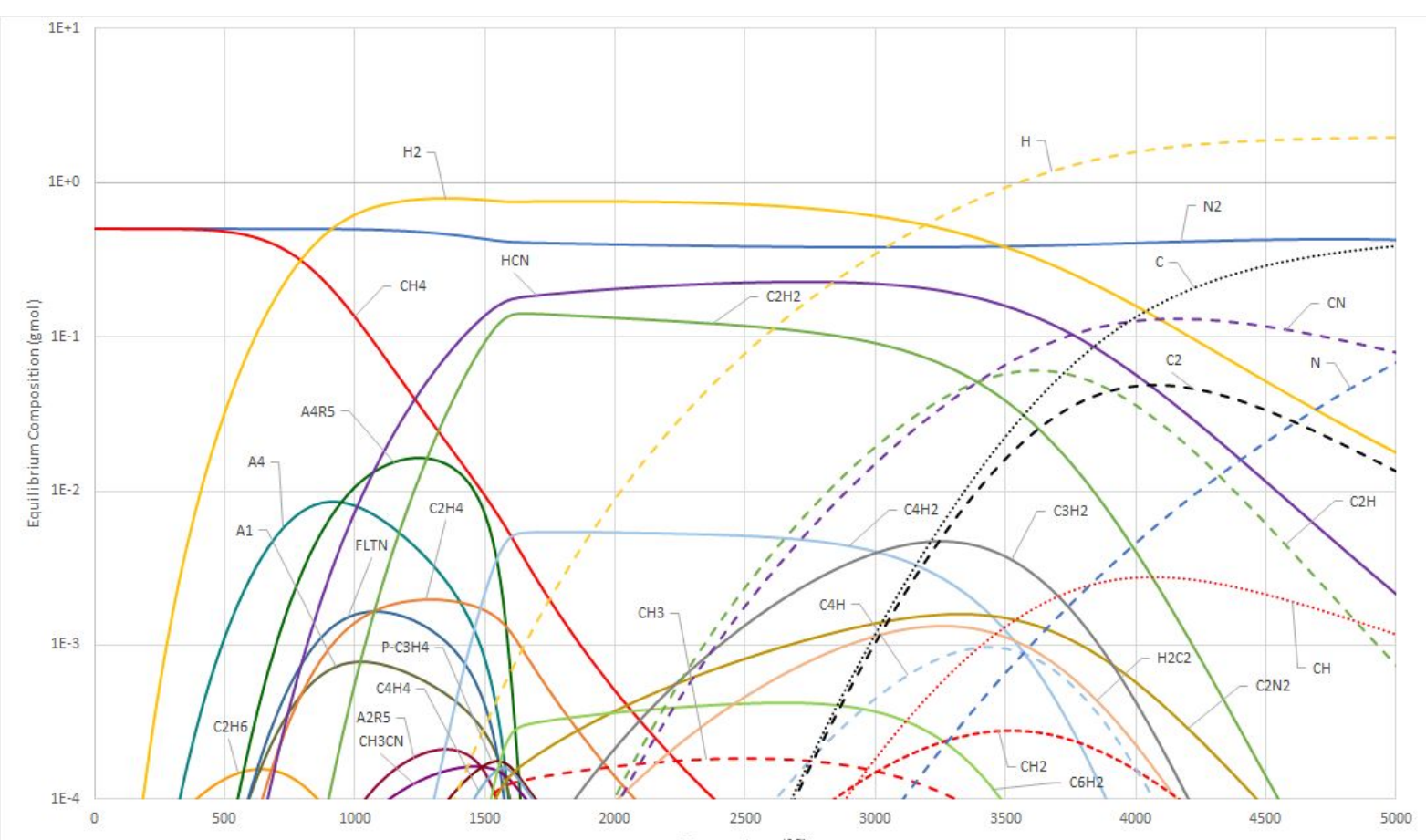

Figure SI-F1 Detailed Thermodynamic profile of C-N-H species (1:2:4), e.g. feed of nitrogen and methane 


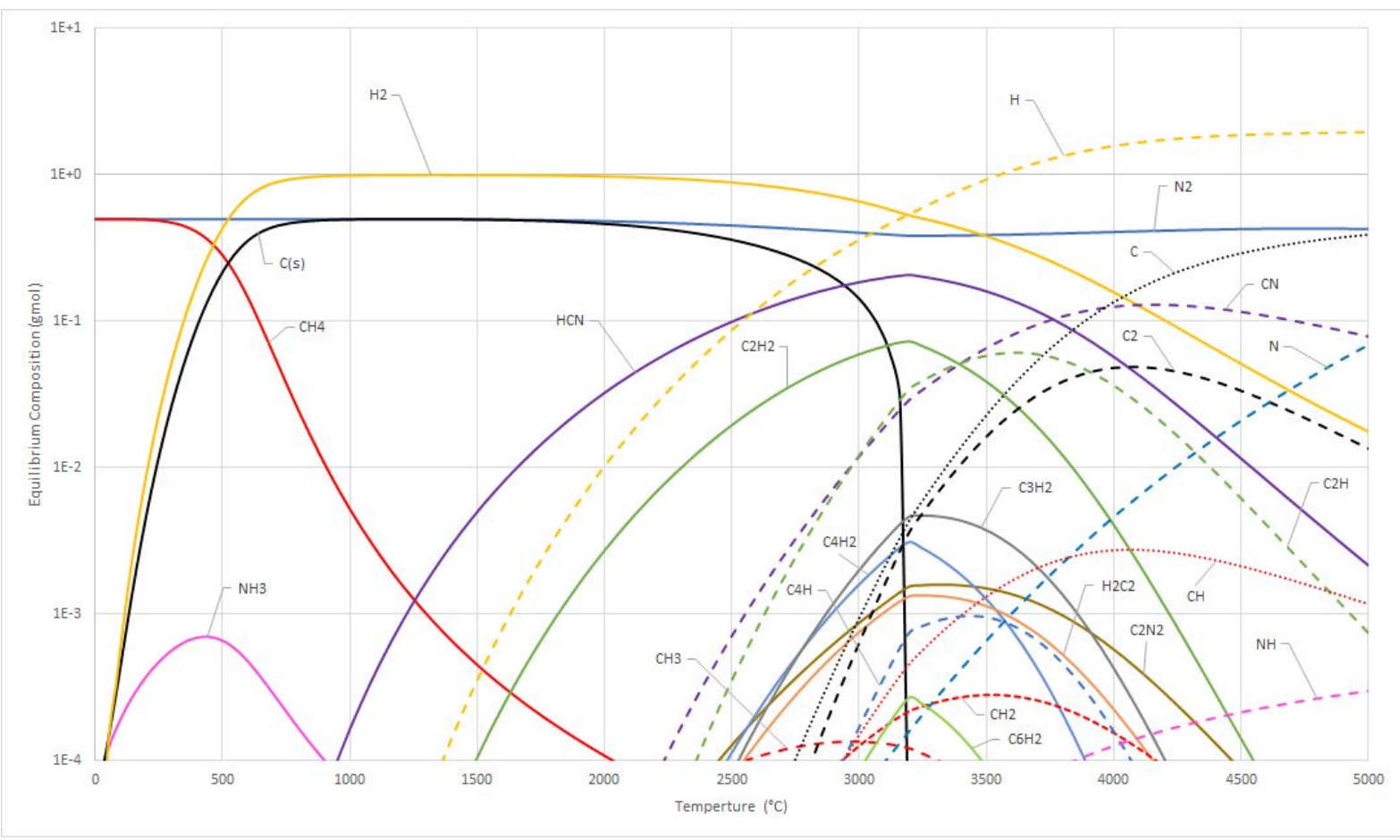




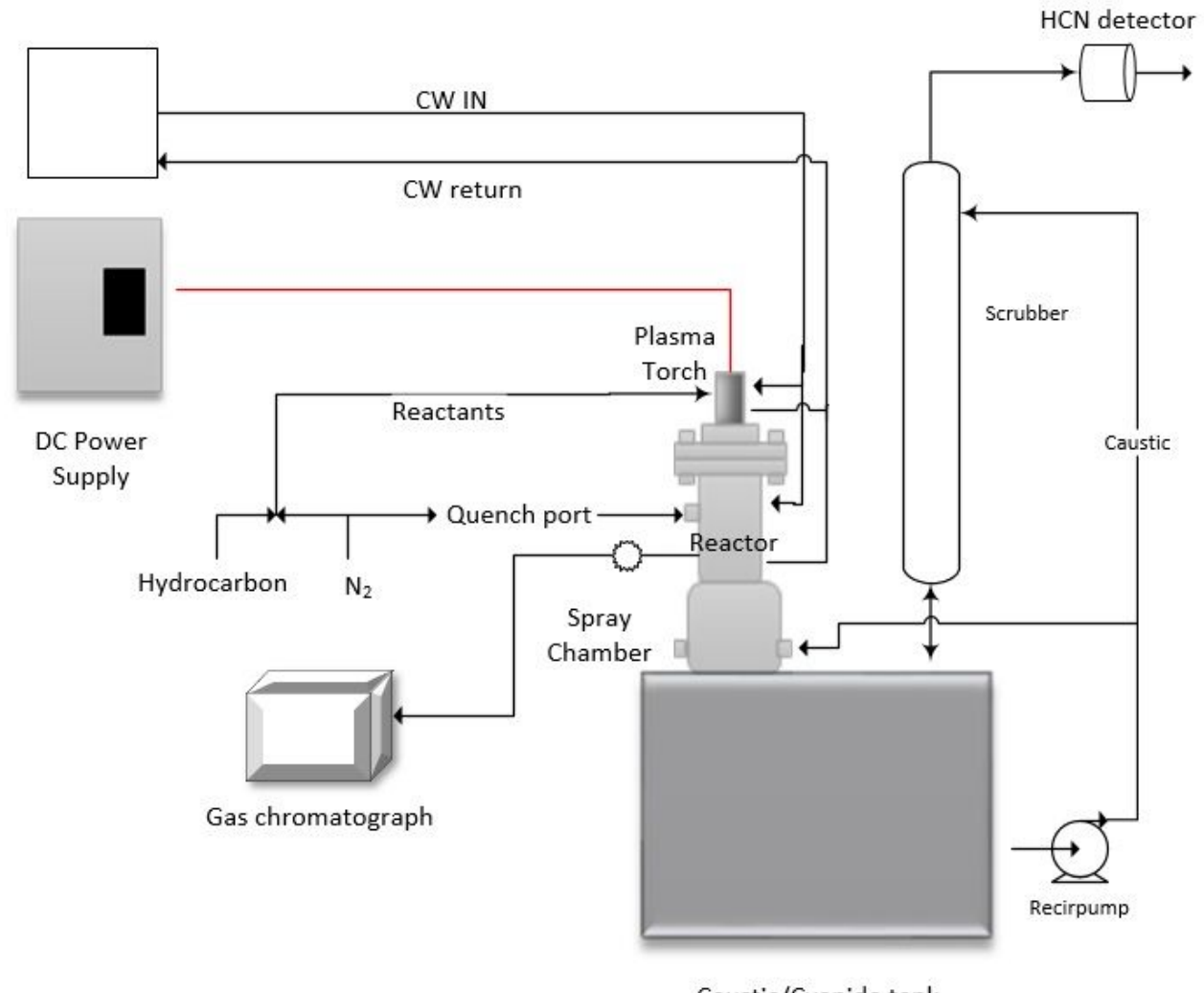

Figure SI-F3: Process flow diagram 
Table SI-T1: Experimental parameters and mass balance - Nitrogen and Propane

\begin{tabular}{|c|c|c|c|c|c|c|c|c|c|c|c|c|c|}
\hline $\begin{array}{l}\text { Test } \\
\text { No }\end{array}$ & $\begin{array}{l}\text { Nitrogen } \\
\text { Flow }\end{array}$ & $\begin{array}{l}\text { Propan } \\
\text { e Flow }\end{array}$ & $\mathrm{N}: \mathrm{C}$ & \begin{tabular}{l|} 
Nitroge \\
$\mathrm{n}$ Mol \%
\end{tabular} & Power & HCN yield & $\begin{array}{l}\text { Cyanide } \\
\text { conv }\end{array}$ & SEC (NaCN) & N2 & C3H8 & Carbon $* *$ & $\begin{array}{l}\text { Carbon to } \\
\text { Acetylene }\end{array}$ & $\begin{array}{l}\text { Carbon to } \\
\text { Soot }\end{array}$ \\
\hline & SLPM & SLPM & & $\%$ & kW & $\mathrm{g} / \mathrm{min}$ & $\%$ & kw-hr/Kg & $\begin{array}{l}\mathrm{mol} / \mathrm{m} \\
\text { in }\end{array}$ & $\mathrm{mol} / \mathrm{min}$ & $\mathrm{mol} / \mathrm{min}$ & & \\
\hline 1 & 46.5 & 7.75 & 4.00 & $85.7 \%$ & 23 & 9.25 & $37 \%$ & 22.8 & 1.88 & 0.31 & 0.94 & $36 \%$ & $27 \%$ \\
\hline 2 & 51 & 6.25 & 5.44 & $89.1 \%$ & 23 & 9.67 & $47 \%$ & 21.8 & 2.06 & 0.25 & 0.76 & & \\
\hline 3 & 64 & 7 & 6.10 & $90.1 \%$ & 23 & 11.44 & $50 \%$ & 18.5 & 2.58 & 0.28 & 0.85 & & \\
\hline 4 & 64.25 & 6 & 7.14 & $91.5 \%$ & 23 & 13.57 & $69 \%$ & 15.57 & 2.59 & 0.24 & 0.73 & $19 \%$ & $12 \%$ \\
\hline 5 & 63.5 & 4 & 10.58 & $94.1 \%$ & 23 & 9.70 & $74 \%$ & 21.8 & 2.56 & 0.16 & 0.48 & $16 \%$ & $10 \%$ \\
\hline 6 & 64 & 4 & 10.67 & $94.1 \%$ & 23 & 9.73 & $74 \%$ & 21.7 & 2.58 & 0.16 & 0.48 & & \\
\hline 7 & 63.5 & 2 & 21.17 & $96.9 \%$ & 23 & 5.19 & $79.45 \%$ & 40.8 & 2.56 & 0.08 & 0.24 & $12 \%$ & 9\% \\
\hline 8 & 57.5 & 8 & 4.79 & $87.8 \%$ & 27 & 12.27 & $47 \%$ & 20.2 & 2.32 & 0.32 & 0.97 & & \\
\hline 9 & 64 & 9 & 4.74 & $87.7 \%$ & 27 & 13.16 & $45 \%$ & 18.8 & 2.58 & 0.36 & 1.09 & & \\
\hline 11 & 72 & 8.9 & 5.39 & $89.0 \%$ & 27 & 15.30 & $53 \%$ & 16.2 & 2.91 & 0.36 & 1.08 & & \\
\hline
\end{tabular}




\begin{tabular}{|c|c|c|c|c|c|c|c|c|c|c|c|c|c|}
\hline 12 & 72 & 9 & 5.33 & $88.9 \%$ & 27 & 16.06 & $55 \%$ & 15.4 & 2.91 & 0.36 & 1.09 & & \\
\hline 13 & 72 & 10 & 4.8 & 87.8 & 27 & 12.40 & 37.93 & 19.99 & 2.91 & 0.41 & 1.25 & & \\
\hline 14 & 32 & 1 & 21.3 & $97.0 \%$ & 14.5 & 2.42 & $74 \%$ & 55.0 & 1.29 & 0.04 & 0.12 & $15 \%$ & $11 \%$ \\
\hline 15 & 32 & 2 & 10.6 & $94.1 \%$ & 14.5 & 4.1 & $63 \%$ & 32.5 & 1.29 & 0.08 & 0.24 & & \\
\hline 16 & 32 & 3 & 7.1 & $91.4 \%$ & 14.5 & 7.17 & $73 \%$ & 18.57 & 1.29 & 0.12 & 0.36 & $19 \%$ & $8 \%$ \\
\hline 17 & 33.5 & 4.2 & 5.3 & $88.9 \%$ & 14.5 & 6.62 & $48 \%$ & 20.1 & 1.35 & 0.17 & 0.51 & $35 \%$ & $17 \%$ \\
\hline 18 & 32 & 4.2 & 5.1 & $88.4 \%$ & 14.5 & 6.57 & $48 \%$ & 20.3 & 1.29 & 0.17 & 0.51 & $34 \%$ & $18 \%$ \\
\hline
\end{tabular}

* The carbon (soot) production was not measured experimentally. It is determined by the mass balance after deducting moles of acetylene and cyanide produced from total carbon supplied. Almost 100\% propane was consumed since no major peak was observed in the product analysis. Trace amounts

37 (<10ppm) of benzene was detected. A few small peaks of long chain compounds were found but not characterised 


\begin{tabular}{|c|c|c|c|c|c|c|c|c|c|c|c|}
\hline Test No & Nitrogen Flow & $\begin{array}{l}\text { Propane } \\
\text { Flow }\end{array}$ & $\mathrm{N}: \mathrm{C}$ & $\begin{array}{l}\text { Nitrogen Mol } \\
\%\end{array}$ & Power & $\mathrm{HCN}$ yield & Cyanide conv & SEC (NaCN) & N2 & $\mathrm{CH} 4$ & Carbon $* *$ \\
\hline & SLPM & SLPM & & $\%$ & kW & $\mathrm{g} / \mathrm{min}$ & $\%$ & kw-hr/Kg & $\begin{array}{l}\mathrm{mol} / \mathrm{mi} \\
\mathrm{n}\end{array}$ & $\begin{array}{l}\mathrm{mol} / \mathrm{mi} \\
\mathrm{n}\end{array}$ & $\begin{array}{l}\mathrm{mol} / \mathrm{mi} \\
\mathrm{n}\end{array}$ \\
\hline M1 & 64 & 19 & 6.74 & $77.1 \%$ & 25 & 12.2 & $59.5 \%$ & 18.7 & 2.58 & 0.77 & 0.77 \\
\hline M2 & 64 & 17 & 7.53 & $79 \%$ & 25 & 12.07 & $65 \%$ & 19 & 2.58 & 0.69 & 0.69 \\
\hline M3 & 64 & 17 & 7.53 & $79 \%$ & 25 & 12.1 & $65 \%$ & 19 & 2.58 & 0.69 & 0.69 \\
\hline M4 & 64.75 & 13 & 9.96 & $83 \%$ & 25 & 11.57 & $82 \%$ & 19.75 & 2.61 & 0.52 & 0.52 \\
\hline M5 & 65 & 14 & 9.15 & $82 \%$ & 26 & 12.95 & $85 \%$ & 19.13 & 2.85 & 0.62 & 0.62 \\
\hline M6 & 64 & 10 & 13 & $87 \%$ & 25 & 9.21 & $85 \%$ & 24.92 & 2.90 & 0.44 & 0.44 \\
\hline M7 & 64 & 7.5 & 17 & $90 \%$ & 25 & 7.15 & $87 \%$ & 32.12 & 2.90 & 0.33 & 0.33 \\
\hline M8 & 64 & 7.5 & 17 & $90 \%$ & 25 & 6.82 & $85 \%$ & 34.96 & 2.85 & 0.33 & 0.33 \\
\hline M9 & 64 & 5 & 25.5 & $93 \%$ & 25 & 5.04 & $92 \%$ & 45.62 & 2.85 & 0.22 & 0.22 \\
\hline
\end{tabular}

$46 * *$ The carbon (soot) production was not measured experimentally. It is determined by the mass balance after deducting moles of acetylene and cyanide

47 produced from total carbon supplied. 100\% methane was consumed since no major peak was observed in the product analysis. 